\title{
The Social Structure of 'Experimental" Strings at Fermilab; A Physics and Detector Driven Model
}

\author{
Mark Bodnarczuk \\ Fermi National Accelerator Laboratory \\ P.O. Box 500 \\ Batavia, Illinois 60510
}

December 12, 1990 
The Social Structure of "Experimental" Strings at Fermilab;

A Physics and Detector Driven Model

\author{
Mark Bodnarczuk \\ Fermi National Accelerator Laboratory \\ P.O. Box 500 \\ Batavia, Illinois 60510
}

\begin{abstract}
Physicists in HEP have been forced to organize large scientific projects without a well defined organizational or sociological model to guide them. In the absence of such models, what structures do experimentalists use to develop social structures in HEP? In this paper, I claim that physicists organize around what they know best, the physics problems they study and the detectors and devices they study them with. After describing the advent of "management" in HEP, I use a case study of 4 Fermilab experiments as the base upon which to propose a physics and detector driven model of social structure for experiments. In addition, I show how this model can be extended to describe "strings" of experiments, where continuities of physics interests, spectrometer design, and a core group of physicists become a definable sociological unit that can exist for over 15 years. A dominate theme that emerges from my analysis is the conscious attempt on the part of experimenters to remove the undertainties that are a part of the practice of HEP. 1
\end{abstract}

\title{
The Advent of "Management" in HEP
}

In the days of cosmic ray experiments, the world was a laboratory in which experimenters set up their detectors in the path of incident particle beams from stellar activities in deep space. But the particle flux from cosmic rays could not be predicted and long detector exposures on high mountain tops (or upon ships) made it difficult to accumulate large samples of particle interactions. ${ }^{2}$ Early "machine builders" designed and constructed accelerators for the purpose of freeing

1 Some of the ideas presented in this paper were presented in the form of an invited talk given at Lawrence Berkeley Laboratory on July 10, 1990. The vast majority of the material is part of my on-going Ph.D. dissertation research at the University of Chicago under the direction of Daniel Garber and William Wimsatt of the Philosophy Department and Bruce Winstein of the Enrico Fermi Institute. In addition, continuing discussions with Lillian Hoddeson, Catherine Westfall, Adrienne Kolb, and Kyoung Paik under the auspices of the Fermilab History Collaboration have been very helpful in formulating some of my ideas.

2 See H. Victor Neher, "Some Reminiscences of the Early Days of Cosmic Rays" in Laurie Brown and Lillian Hoddeson (eds.), The Birth of Particle Physics, (New York: Cambridge University Press, 1986) pp $120 \mathrm{ff}$, and Carl D. Anderson and Herbert L. Anderson, "Unraveling the Particle Content of Cosmic Rays," in Laurie Brown and Lillian Hoddeson (eds.), The Birth of Particle Physics, (New York: Cambridge University Press, 1986) pp $131 \mathrm{ff}$. 
experimentalists from the uncertainties inherent in performing cosmic ray studies. ${ }^{3}$ The ability to produce particle beams of a defined energy and intensity at will enabled them to be free of the intermittent cosmic ray particle beams and accumulate much larger data samples.

With the advent of particle accelerators, experimentalists began setting up their detectors at particle accelerator laboratories in the path of incident particle beams produced by accelerators. In the 1950's, accelerators had circumferences of order 63-126 meters and could be housed in large buildings. ${ }^{4}$ With the discovery of strong focusing by Courant, Livingston, Hartland, and Snyder, the size of accelerators in the 1960 's grew to have circumferences of order 628 meters and were housed underground in tunnels. ${ }^{5}$ Machines in the 1970's (like Fermilab's Main Ring) were about 6 kilometers in circumference, reaching an energies between 200.400 $\mathrm{GeV}$, and with the advent of the superconducting Tevatron, reached energies of 1.8 $\mathrm{TeV}$ in the center of mass in the early to mid $1980^{\prime} \mathrm{s}^{6}$ In the mid to late 1980 's, accelerators like the Large Electron Positron Collider (LEP) at CERN grew to about 27 kilometers in circumference, and the SSC (designed for operation in the next millennium), has a circumference of about 90 kilometers and will reach energies of $40,000 \mathrm{GeV}$ in the center of mass. $^{7}$ Because the required circumference of the accelerator is approximately inversely proportional to the B-field strength of the magnets used in the machine, the rising masses of the particles to be studied and the falling cross sections for particle production demanded the size and complexity of the machines built also increase. But rising particle masses and falling cross sections for particle production also meant a substantial rise in the cost of building these larger and larger machines. Thus, the resources for doing HEP research were localized in a few institutional laboratories, creating a monopoly that came to be funded largely by the U.S. Government. 8 The claim that the size, complexity, and cost of these

\footnotetext{
3 Heilbron and Seidel claim that the main motivation of early "cyclotroneers" like Lawrence was the "macho character" of building bigger and bigger machines and were only secondarily concerned about the experiments done with those machines. See J. L. Heilbron and Robert W. Seidel, Lawrence and His Laboratory; A History of the Lawrence Berkeley Laboratory, vol. 1 (Berkeley, CA: University of California Press, 1989), p 466.

4 The Cosmotron at BNL had a beam energy of $3 \mathrm{GeV}$, while the Bevatron at the Lawrence Radiation Laboratory could reach energies of $6.2 \mathrm{GeV}$.

5 The Alternate Gradient Synchrotron at Brookhaven National Laboratory could reach energies of about $33 \mathrm{GeV}$, the CERN Proton Synchrotron about $28 \mathrm{GeV}$, and the "weak" focusing Zero Gradient Synchrotron at Argonne National Laboratory could reach energies of about $13 \mathrm{GeV}$. See Laurie M. Brown, Max Dresden, and Lillian Hoddeson, "Pions to Quarks: Particle Physics in the 1950's" in Laurie M. Brown, Max Dresden, and Lillian Hoddeson (eds.) Pions to Quarks; Particle Physics in the 1950's, Based on a Fermilab Symposium, (New York: Cambridge University Press, 1989), p 13 ff.

6 The Fermilab Main Ring was originally designed as a $200 \mathrm{GeV}$ machine that later ran at $400 \mathrm{GeV}$. See Catherine Westfall's, The First "Truly National Laboratory": The Birth of Fermilab, 2 vols, unpublished dissertation submitted to Michigan State University, Department of English, 1988, vol 1, p $160 \mathrm{ff}$.

7 My primary focus here is the size of accelerators, not the particle type, thus $I$ have included LEP in what is otherwise a description of hadron colliders.

$8 \mathrm{~J}$. L. Heilbron and Robert Seidel discuss how the development of accelerators was inextricably tied to government funded weapons research. See J. L. Heilbron, "An Historians Interest in Particle Physics," in Laurie M. Brown, Max Dresden, and Lillian Hoddeson (eds.) Pions to Quarks; Particle Physics in the 1950's, Based on a Fermilab
} 
accelerators was powerfully driven by the need to penetrate into new physics domains is clear. But what is much less clear is that the size, complexity, and cost of these machines also demanded that more complex sociological structures be developed to manage the monopoly that these laboratories had on the resources for doing HEP.

The ability to produce particle beams of a defined energy and intensity at will enabled experimentalists to be free of the uncertainties of naturally produced cosmic rays, but this thrust them into a new type of sociological uncertainty produced by the monopoly that accelerator laboratories had on particle beams. For scientists in HEP, such social factors are sometimes more difficult to navigate than the capriciousness of incident cosmic ray particles. My claim is that the rise of the institutional monopoly on resources gave birth to the large scale "management" of HEP. The monopoly on resources subsequently produced 3 new and powerful sociological factors: proton economics, experimental real estate, and a redefinition of what it meant to be a particle physicist in HEP. The first such factor, proton economics, was vitally constrained by the physical parameters of the accelerator's ability to produce a given number of protons and the cross section for particle production. The sociological and management parameters emerged when considering how to best utilize these resources, namely the choice of which types of particles beams to produce and how to distribute them among the experimental community at a laboratory like Fermilab.

The physical parameters of proton economics involved a number of constraints, the first of which was the cross section for secondary and tertiary beam production. Given the total number of protons from the accelerator, the Laboratory had to decide what type of secondary beams to provide for experimenters in light of the experimental proposals that were submitted. For example, because the cross section for neutrino production is $10^{-36} \mathrm{~cm}^{2}$ and the pion cross section is $10^{-27} \mathrm{~cm}^{2}$ the decision to approve experiments that used incident beams of neutrinos was already a major decision which affected proton economics. ${ }^{9}$ It was clear that a neutrino beam was much more costly than a pion beam in terms of the number of protons needed, especially if the intensity of the secondary beam was of large order.

The second constraint of proton economics was the cross section for particle production in the experimental target. The decision to propose an experiment had to be proceeded by a monte-carlo study of how many potential events could be detected in the experimental spectrometer with a given secondary beam intensity. By knowing the secondary beam intensity, the repetition rate, the physical properties of the target (luminosity), and the cross section for the interaction to be studied, the experimenters could calculate a reaction rate for a particular type of particle event. Once the reaction rate was calculated, the experiment determined the number of events needed to provide adequate statistics in their data sample, given a specific number of beam hours. When proposing an experiment, the collaboration began by calculating the desired reaction rate and working back through the process to

Symposium, (New York: Cambridge University Press, 1989), pp $50 \mathrm{ff}$, and Robert Seidel, "Postwar Political Economy of High-Energy Physics," in Laurie M. Brown, Max Dresden, and Lillian Hoddeson (eds.) Pions to Quarks; Particle Physics in the 1950's, Based on a Fermilab Symposium, (New York: Cambridge University Press, 1989), pp $497 \mathrm{ff}$.

9 The pion cross-section is roughly constant for energies above $2 \mathrm{GeV}$ at about 40 millibarns. The neutrino cross section is not constant but is linearly proportional to the energy. For Fermilab, a reasonable neutrino energy to use is $100 \mathrm{GeV}$, which would give a cross section of about 0.7 picobarns. I would like to thank Anthony Malensek of the Fermilab Research Division for his input here. 
determine the number of incident protons needed on the primary production target and included this number as part of the experimental proposal. 10

The rise of the institutional monopoly on the resources to do HEP led to a second powerful sociological factor, experimental real estate, which was related to proton economics but was somewhat less defined. Given the size and complexity of experimental detectors and the long lead times needed to assemble, operate, and analyze the data, collaborations of physicists who were given beam time moved into the experimental hall at the end of one of the particle beam spigots with the explicit goal of not moving out. The experimental proposals were based upon the collaboration using general purpose equipment provided by the laboratory. Only portions of the spectrometer were built using the resources of the collaborating universities themselves. In some instances, the laboratory invested large amounts of resources into more general purpose facilities that (with minor modifications) could be used to do a series of different experiments. 11

The phenomenon of experimental real estate manifested itself in a number of ways, all of which were aimed at not moving out of the enclosure once a collaboration has secured that real estate. Incumbent experiments could try to convince the laboratory of the importance of the potential physics with the goal of securing more laboratory resources for a follow-up experiment. The resources were not just beam time, but often involved the resources needed to upgrade the spectrometer. If they succeeded, they could leave the major components of their spectrometer in place and gain additional access to the beam. If the above strategy failed and other experimental groups threatened to displace them by submitting a proposal which used that beamline and enclosure, the next best strategy was for the incumbent experiment to try to convince the laboratory (and the intruding experimenters) to use portions of their spectrometer as part of the intruding experiment's spectrometer configuration (something which the competing group argued against). Doing this enabled the incumbent to partially "hold his place in line," and increased the probability that the laboratory would allow them to do subsequent experiments. In generating their arguments against giving up the experimental real estate, the incumbent experiment would use the data from their previous run and the promise of new results to show that their experiment was "more important" to the progress of HEP than the proposed results of the experiment seeking to displace them.

The rise of the institutional monopoly on the resources to do HEP produced a third sociological factor which involved a redefinition of what it meant to be a physicist in the discipline of HEP. This manifested itself as an increasingly complex division of labor where the major responsibility of some physicists was to provide the laboratory support which allowed other physicists to perform experiments. These "service" jobs involved activities like the design, construction, and operation of the accelerators that produced the incident beams, the external beamlines used to

10 For example, a rough summation of all the intensity requirements for experiments proposing run during the 1993 fixed-target run at Fermilab is about $5 \times 10^{13}$ protons. The accelerator is currently capable of producing about $1.5 \times 10^{13}$ protons. The parameters of proton economics will vitally constrain the total number of proposed experiments will actually be approved to run.

11 An early example that $I$ will focus on as a case study, is the Tagged Photon Magnetic Spectrometer at the Tagged Photon Lab. But with Leon Lederman's decision to propose Tevatron II (an upgrade of the fixed-target experimental areas to transport $800 \mathrm{GeV}$ beams which included the construction of a number of large experimental facilities), Fermilab began to place more emphasis on building general purpose spectrometers that could be used to exploit an entire program of physics experiments. 
transport those beams to the experimental apparatus, and the computing support needed to analyze the data from experiments. Thus the purpose of the physicists which constituted this new support structure was to make the resources of HEP available, but to whom?

There were two directions that this took depending on the ideologies of a particular laboratory. At laboratories like the Brookhaven National Laboratory (BNL) and the Lawrence Berkeley Laboratory (LBL), the strong "in-house" groups which maintained these systems also exerted control over the resources, making it more difficult for "outside" users to gain access to the particle beams. Within the physical parameters of proton economics, laboratory management decided to distribute the particles beams to users who were affiliated with the laboratory institution. Thus there was little or no distinction between the sociological structure that made the resources of HEP available, and the sociological structure of the experimenters that utilized those resources. At Fermilab, there was a much more pronounced division between those who provided the resources and the outside users that wanted to gain access to the beams. The redefinition of what it meant to be a physicist in HEP was seen most profoundly at Fermilab because the in-house/outside-user distinction was built so deeply into Robert Wilson's image of laboratory life. 12 With the in-house style of management, affiliation with the institution was the best strategy for securing resources. With the Fermilab style of management, it was more important to convince laboratory management that the physics was worth doing and that the collaboration had the expertise and manpower to actually perform the experiment. While the "philosophical" differences between the two management approaches are markedly different, they are clear examples of the increasingly complex social structure and management needs of HEP. Unlike the marked absence of such factors in cosmic ray experiments, navigating the social negotiations involved in proton economics and experimental real estate became at least as important as anything "experimental" in the actual process of beginning, operating, and ending an experiment.

\section{A Physics and Detector Driven Sociology: a Case Study}

Having described the emergence of the higher-level sociological structures needed to manage the resources of HEP at the institutional level, I will now focus upon the less understood lower-level issues of the social structure of the experiments that utilize those resources. I will attempt to show that within the context of the experiment too, sociological factors can be at least as important as anything "experimental" when performing an experiment. The central claim of this section is that because there is no systematic sociological model upon which to structure collaborations, they organize themselves around what they know best, the physics problems they study and the spectrometers they study them with. I will show how a definable method for analyzing the social structure of collaborations emerges from a description of their physics goals and a diagram of their spectrometer and is what I refer to as a physics and detector driven sociology for experiments. To substantiate this claim, initially, I will briefly discuss the history of the development of detectors,

\footnotetext{
12 Wilson was committed to what Lederman called a "TNL," a truly national laboratory, the acronym of which was a phonetic play on BNL where in-house groups dominated the resources for doing HEP. As Wilson remarked, "...NAL leaders intended to make outside users "the whole focus" of the laboratory, in keeping with the spirit of Lederman's "Truly National Laboratory." See Westfall, p 331 ff.
} 
then use the example of experiment E-516 (Photoproduction of Charm) and other experiments which ran at Fermilab.

The history of the development of the detectors used for HEP moved along two definable but orthogonal axes, both of which were the direct result of the nature of the physics problems being studied. Along the first axis, detectors increased in size, but not necessarily in architectural complexity. An example highlighted by Peter Galison, was the development of the table-top sized spark chamber by Cronin and Renninger in 1960, and its expansive extrapolation by Melvin Swartz and Leon Lederman into a device that was large enough to be sandwiched between surplus naval cruiser deck plates weighing between two and three thousand tons. 13 The extrapolation from spark chambers that were table sized to the mammoth detectors used by Lederman in the two-neutrino experiment performed at BNL or the Fermilab based E-1A experiment performed by Rubbia, Cline, and Mann was necessitated by the fact that they were both performing studies on neutrino interactions. It was the cross section for neutrino production that necessitated the larger target mass along with the increased requirements for shielding the fiducial volume of the detector. Consequently, the design of such experiments was inextricably tied to the physical properties of the neutrino cross section and the cross sections of the hadrons and leptons that composed the background for weak neutral current events. ${ }^{14}$ But because detecting neutral currents only involved detecting the presence or absence of a muon track protruding from the cluster of hadrons produced in the incident neutrino collision, the E-1A detector (for all its size) was a relatively uncomplex detector having only 46 spark chambers attached to 46 channels of electronics. Growth in the size of the detectors also meant an increase in the cost of experiments like E-1A. This made collaborations more than ever dependent upon the laboratory for the resources needed to construct these mammoth structures.

In the orthogonal axis of complexity, many varieties of detector types were combined to form increasingly complex spectrometers composed of a variety of detector sub-systems. These complex spectrometers enabled experimentalists to record more complex physical interactions and measure increased numbers of physical parameters simultaneously. The Tagged Photon Magnetic Spectrometer (TPMS) used for experiment E-516 at Fermilab was an early example of a spectrometer which grew substantially along both axes, being large in size as well as complexity. 15 The goal of the experiment was to study photoproduction of final states of mass above $2.5 \mathrm{GeV}$. The spectrometer was an array of individual detector sub-systems that were designed to function as a unit, summed at a higher level by a specially designed computing architecture. 16 Because the decay products of charmed particles were more complex than the binary requirement of the E-1A detector, the measurement needed to reconstruct the multiple final states demanded that the TPMS be a significantly more complex apparatus. It was divided into 6 detector regions; the

13 Peter Galison, "Bubbles, Sparks, and the Postwar Laboratory," in Laurie M. Brown, Max Dresden, and Lillian Hoddeson (eds.) Pions to Quarks; Particle Physics in the 1950's, Based on a Fermilab Symposium, (New York: Cambridge University Press, 1989), pp 235-237.

14 An careful account of the E-1A experiment has been written by Peter Galison, How Experiments End, (Chicago: The University of Chicago Press, 1987).

15 See the Tagged Photon Magnetic Spectrometer; Facility Design Report, May 9, 1977. Other examples at Fermilab were the Multi Particle Spectrometer used for experiment E557 and the spectrometer used for experiment E-400 in the Broad Band Photon Beam Line.

16 Experiment E-516 used a ECL trigger processor that was specially designed by Tom Nash and Steve Bracker. The ECL trigger processor was described in an article written in the May, 1983, issue of Physics Today. 
experimental target/recoil detector, the tracking system, the Cerenkov counter, the segmented liquid scintillation shower counter (SLIC), the hadromagnetic calorimeter, and the muon system. ${ }^{17}$ The complexity of the particle interactions was mirrored in the complexity of the spectrometer which had 7,000 interaction points distributed over the 6 detector regions, each of which was connected to a channel of electronics. 18 What this points to is a strong causal relationship between the complexity of the physics interactions and the complexity of the detectors needed to detect those interactions. It also points to the fact that the cost of building such spectrometers substantially increased by virtue of its size and by virtue of its complexity, because more channels meant more money. ${ }^{19}$ When spectrometers became as large and complex as the TPMS, the institutions that composed the collaboration not only became more dependent upon the laboratory for financial resources, they also became more interdependent upon one another for bearing their share of the cost of the experiment. The comparison of the E-1A experiment and experiment E-516 points to a physics driven detector design. For those who are familiar with the scientific practice of HEP, this conclusion ought not be surprising.

What may be surprising is that the physics driven explanation of detector designs does not stop at the detectors but extends to the sociological structure of the collaboration itself. In the absence of any systematic sociological model for organizing the E-516 collaboration, the physical structure of the TPMS became the model upon which the social structure of the collaboration was formed. Each of the 6 regions of the spectrometer was the domain of a particular institutional group from the collaboration and represented their hardware contribution to the overall experiment. The recoil detector was built by the University of Toronto, the SLIC was built by the University of California at Santa Barbara, the Cerenkov counters were built by the University of Colorado at Boulder, and the tracking system, hadronic calorimeter, and muon system were built by Fermilab scientists. 20

In other words, the basic social structure of experiment E-516 emerges from the drawing of the TPMS when the universities and institutions responsible for the design and construction of the detector sub-systems are identified. Add to this the fact that each of these institutions had an institutional representative, and the basic social structure of E-516 begins to take shape. The complexity of the physics interactions determined the physical structure of the TPMS, and the people who planned to carry out the experiment organized (taxonomized) themselves around this structure by institution and area of expertise. As spectrometers like the TPMS were carved up into smaller, more manageable pieces, the phenomenon of "technical specialization" emerged in a far more powerful way. The sociologist of science

17 See the diagram of the E-516 configuration of the TPMS in the Tagged Photon Magnetic Spectrometer; Facility Design Report, May 9, 1977.

18 Physicists normally refer to an "interaction" point as a point in the experimental target. What I am referring to are points within the detector sub-systems where the decay products from particles produced in the experimental target interact, for example, the cell of a drift chamber, or the wire of a PWC.

19 A rough rule of thumb for estimating the cost of a spectrometer is to quantify cost in terms of dollars per channel. In a private communication with Ken Stanfield who designed the drift chambers for the E-516 tracking system, Stanfield claimed that one of the major factors in determining the number of wires/channels that would constitute the tracking system was dividing the dollar amount of the E-516 budget for tracking by the cost per channel.

20 The ECL trigger processor and the on-line computing system were collaborative efforts between Fermilab and the University of Toronto contingents of the collaboration. 
seeking to understand the most fundamental aspect of the social structure of an experiment like E-516 must begin the analysis with a diagram of the apparatus itself. In a well defined way, the complexity of the social structures of collaborations emerge from the complexity of the spectrometer, which in turn is driven by the complexity of the physics interactions to be detected. The descriptive ability of the physics and detector driven model can be extended even further to describe all of the phases of the experiment and reconstructing and analyzing the data. It even extends to the actual production of the scientific papers of E-516.

In the design, construction, and installation phase, the physical and financial demands of getting the spectrometer built and operating forces the collaboration members to focus more of their attentions upon that region of the spectrometer for which they are responsible. Because many of the detector sub-systems were constructed at the home institutions then shipped to Fermilab for installation, there tended to be less social interaction between the the members of the collaboration in the earlier phases of the experiment. 21 The level of social interaction of the experiment was significantly increased when they come together as a group at the laboratory during the installation phase of the experiment. From this point on, the social interaction of the collaboration continued to increase during the actual data taking phases of the experiment. But it seems clear that in addition to the TPMS being the basis upon which the social structure of the collaboration was formed, it was also the determining factor in the magnitude of the social interaction.

During the running of the experiment, the sociological taxonomy of the detector sub-systems defined the way that technical problems and challenges were addressed by the collaboration. Problems in the operation of the SLIC were within the domain of the Santa Barbara contingent of the collaboration who designed and constructed the detector, whereas problems with the Cerenkov counters were considered the purview of the University of Colorado part of the collaboration. To be sure, various members of the collaboration gained a limited knowledge of the detector sub-systems for which they had no direct responsibility, but the major repositories of expertise for running the experiment were taxonomized and distributed along the lines shown in the diagram of the TPMS. 22

The reconstruction phase of the experiment was spread over a two year period which spanned 3 distinct data runs. ${ }^{23}$ During this phase, there was a relaxing of the well defined distribution of expertise as various members who had responsibility for one specific detector sub-system developed reconstruction packages for other regions of the TPMS. In fact there were multiple reconstruction packages written a each of the TPMS detector sub-systems. ${ }^{24}$ During the reconstruction phase of the experiment the boundaries that organized the collaboration around the spectrometer were more easily crossed as one member of the collaboration attempted to extend his

21 There were of course design meetings and reviews performed both within the collaboration and in the context of the laboratory, but the actual detector components were often constructed at the home institution and subsequently shipped to Fermilab.

22 The problems associated with all members of a collaboration not having an in depth knowledge of all parts of a large complex spectrometer due to the need for "technical specialization" are well known to experimentalists in HEP, especially with large collider experiments like CDF.

23 The first shakedown run of E-516 was in the Summer of 1979, with the first data run beginning in the late Fall of 1979. The final data run began in March of 1980 and ended at the accelerator shutdown in June, 1980.

24 For instance, their were 3 separate reconstruction packages generated for the tracking system, and 2 packages generated for the SLIC. This is a common scientific practice in HEP experiments. 
area of expertise to detector sub-systems that were the responsibility of another group in the collaboration. While the foundation of a collaboration's social structure is anchored in the spectrometer design throughout all phases of an experiment, the example of E-516 shows that these taxonomies become less rigid in the later phases of the experiment when tasks involve the medium of software rather than hardware. 25

In the final data analysis and publication phase, the collaboration faced the issue of social negotiation in the production of final publications. But even at this stage, it was the detector that was the focus and major taxonomizing factor of such sociological issues. For example, the primary expertise needed for the calibration of the SLIC were still addressed mainly by Santa Barbara group who designed and built the detector, while the primary expertise for the Cerenkov counter was considered within the domain of the Colorado group. 26 What is interesting sociologically is that in the same way all of the physical components of the TPMS had to work as a unit in order to produce the evidence that would support the data in the publication, the human components that were taxonomized and distributed along the boundaries of the TPMS also were forced to work together as a unit. In the same way the 6 regions of the spectrometer had to work together as a unit in order to detect the decay products of charmed particles, the collaboration had to work together as a unit in order come to agreement on the final form of the publication.

In addition to the social structures that were defined by the TPMS itself, there was a higher-order layer of social structure that is important to describe. The laboratory requires experiments to appoint a spokesperson that is the liaison between Fermilab and the collaboration in all matters to do with the experiment. The "management" style of the spokesperson is also an important element in social negotiation that occurs within the collaboration and between the experiment and the Directorate. Almost without exception, it is the spokesperson and the laboratory Directorate that are the major players in what might be characterized as the Director/experiment relationship. While this higher-order level is important to the "management" of the collaboration, it is far less important to defining the basic social structure of the collaboration. Independent of the management style of the spokesperson (autocratic, democratic, etc.), the underlying social taxonomy of the spectrometer persists as the foundation of higher-order social structures. For instance, shortly after the laboratory's approval of the follow-up experiment to E-516 (E-691) there was a rotation of spokespersons for the collaboration. 27 But the basic social structure of the collaboration remained taxonomized around the physical design of the TPMS throughout this rotation. In addition, all modifications to the E691 configuration of the TPMS were mirrored in the expansion of the responsibility of part of the collaboration. 28 Even in the follow-up experiment, the diagram of the

25 Part of this is due to the inability of the collaboration to secure the resources necessary to construct multiple detector sub-systems. The resources needed to produce software are much easier to obtain because they only involve the time and expertise of the students and physicists on the experiment which are directly under their control and have little or no financial impact.

26 Analysis of the meeting minutes of the collaboration also support this view.

27 Tom Nash, spokesperson for E-516, rotated the position of spokesperson with another member of the E-516 collaboration, Mike Witherell, in order to devote his time to the development of the new low-cost high-power parallel processing computers which he proposed to the laboratory a year earlier. See "A Program for Advanced Electronics Projects at Fermilab" by Tom Nash, May 11, 1982. This proposal eventually developed into the Advanced Computing Project (ACP) under the leadership of Nash.

28 As I will describe in more detail in the next section, the addition of the Silicon Microstrip Detector was the responsibility of the Santa Barbara group. 
spectrometer continues to define the social structure of the collaboration in a powerful way. 29 As I claimed earlier, the complexity of the social structures of collaborations emerge from the complexity of the spectrometer, which in turn is driven by the complexity of the physics interactions to be detected. But the example of E-516 reveals another crucial sociological factor emerging from the spectrometer, that is, the operation of the spectrometer as a unit forcing the collaboration to likewise work together as a unit. The combination of these factors provides the basis for extending the descriptive ability of the physics and detector driven model beyond a single experiment to "strings" of experiments, revealing even more complex and long-lived sociological structures.

\section{An Experimental "String" Theory}

Experiments E-516 and E-691 met at a transition-like interface where the first experiment seemed to transform into a second experiment. 30 Most physicists in HEP understand this process intuitively and can even identify many components of what appears to be a transformation process. But such intuitions have yet to be concretized in any systematic way either by physicists or by those who are interested in characterizing the sociological structure of collaborations in HEP. ${ }^{31}$ A necessary ingredient in concretizing these intuitions about "strings" of experiments, is the ability to demonstrate what the continuities are between the experiments that constitute such "strings." The claim of this section is that the continuities between multiple experiments like E-516, E-691 and their progeny are constituted by the parameters already described in the physics and detector driven model, in combination with the ability of a core group of physicists to work together effectively as a unit in a continuing program of physics studies that yield successful results.

The configuration of the TPMS for E-516 and E-691 were almost identical, but there were a few important differences between them. 32 The most important difference was the use of the Silicon Microstrip Detector (SMD) instead of the recoil detector and more loosely defined trigger assumptions. 33 The SMD allowed E-691 to

29 As I will describe in detail in the next section, the only major structural addition to the TPMS was the Silicon Microstrip Detector which replaced the target and recoil region of the E-516 configuration.

30 Experiment E-691 was formally proposed to the laboratory on February 4, 1981 during the reconstruction and analysis phases of E-516.

31 While Peter Galison provides a veridical account of the role that experiment plays in the development of scientific knowledge in HEP, he is more interested in the issue of "realism" and sociological considerations are beyond the scope of his work. See Peter Galison, How Experiments End, (Chicago: The University of Chicago Press, 1987).

32 Unlike the $400 \mathrm{GeV}$ primary proton beam that serviced E-516, experiment E-691 ran with an incident proton beam of $800 \mathrm{GeV}$ from the newly completed Tevatron. This allowed the photon energy that was incident on the experimental target to be increased from about $160 \mathrm{GeV}$ (for the E-516 configuration) to about $300 \mathrm{GeV}$ (for E-691).

33 Whereas E-516 used the recoil detector in combination with tightly defined trigger assumptions to look for the charm signature of a recoil proton, E-691 used a more loosely defined high $P_{t}$ trigger in combination with the Silicon Microstrip Detector. It is interesting to note that the spectrometer configuration was so similar to the one used for E-516, that the collaboration simply attached a copy of the original Tagged Photon 
reconstruct the decay vertices of charmed particles in the upstream region of the spectrometer and project the tracks of particle decays to the forward regions of the spectrometer. Experiment E-516 triggered the TPMS with the upstream recoil detector and projected the particle tracks back toward a vertex that could not be as carefully defined by the recoil detector. In addition, E-691 made a significant addition to the on-line computing capabilities, using a VAX $11 / 780$ for data monitoring, and the PDP11/55 solely for data acquisition. 34 The use of the SMD and the substantial increase in on-line computing capabilities were the only major changes between the two experimental configurations. ${ }^{35}$ A comparison of the diagrams of the TPMS shows that there was a strong continuity between the two experiments.

In regard to the continuity of physics goals, the original proposal of P-516 already included an ambitious physics program for the spectrometer that transcended the studies done by E-516. The plans for using the TPMS in the E-691 Tevatron configuration were already explicitly stated as early as 1976.36 The proposal states, "In fact, although this is by no means a proposal for the Energy Doubler, this spectrometer will be ideal - and unique - for studying photoproduction when the Doubler comes into operation. It is probably the only existing $P$ East facility that will be able to operate at $1000 \mathrm{GeV}$. The extra proton energy will be used either to increase photon intensity in the $70-140 \mathrm{GeV}$ range or to double the photon energy. The latter would involve no modification of the electron beam which is capable of $300 \mathrm{GeV} . " 37$ When this suggestion in the proposal is compared with the actual history of E-691, it is clear that the outline for what was later submitted as the proposal for E-691 was already anticipated by the collaboration. 38

But the first P-516 proposal also laid out a program of study that extended even beyond the boundaries of E- 691 by saying,

"The electron beam can also be used to transport pions into the Tagged Photon Lab. R. Rubinstein notes that although spot sizes will be somewhat larger the intensities are potentially only a factor of about 3 below the $P$ West pion beam. Thus, one can imagine a future proposal to use the spectrometer at the TPL for direct comparison of photoproduction and hadron production with systematic errors caused by using different detectors eliminated. This emphasizes the

\footnotetext{
Magnetic Spectrometer: Facility Design Report produced for E-516 in 1977 to their proposal for E-691 (Proposal to Do Photon Physics with the Tevatron at the Tagged Photon Spectrometer). This included the same spectrometer diagram used for E-516 and the differences between the two configurations were simply described in the text.

34 For E-516, the PDP11/55 was used for both data acquisition and data monitoring.

35 The collaboration also added 1 additional bank of drift chambers to the forward region of the tracking system which enabled them to have finer resolution in resolving the trajectories of particles that penetrated the tracking system.

36 E-691 was formally proposed in February, 1981 and not approved by the laboratory until November, 1983.

37 The name Energy Doubler was a DOE label attached to what was originally called the Tevatron. See the Proposal to Study Photoproduction of Final States of Mass Above $2.5 \mathrm{GeV}$ with a Magnetic Spectrometer in the Tagged Photon Lab, submitted October 1, 1976 by Jeff Appel, Paul Mantsch, and Tom Nash (Fermilab), Rolly Morrison (University of California, Santa Barbara), and George Luste (University of Toronto), pp 2-3.

38 I have also establish this fact in private communications with Tom Nash, Rolly Morrison, and other members of the E-516/691 collaboration.
} 
flexibility and long range benefits to the Proton East program that the spectrometer we propose would bring." 39

It is with the assertion that the TPL beamline can be converted to a pion beam, enabling the TPMS to be used to do hadronic studies of charm, that the original P-516 collaboration outlines the full program for exploiting what was then only a proposed facility. An analysis of the diagrams of the TPMS configurations for experiments E$516,691,769$, and 791 shows that the majority of the detector sub-systems remained in tact and that all substantial changes were simply additions to (or modifications of) the basic structure of the spectrometer. 40 As described above the core group of physicists from E-516 transformed it into its prodigy, E-691. But an analysis of the names on the proposals for experiments E-769 and E-791 show that there was also a distinct continuity in the core group of physicists that proposed these experiments and the institutions they were affiliated with. 41 As was indicated already in the P+516 proposal, the primary beam was eventually converted to transport pions which enabled the collaboration to study hadroproduction of charmed particles (submitted as the proposal for E-769), and the hadronic decays of charmed particles (submitted as the proposal for E-791). 42

This example shows that there are sociological and experimental structures and continuities that transcend a single experiment and can provide a framework for understanding what appears to be the more complex social structure that spans the 15 year history of these 4 experiments. Each experimental configuration of the TPMS displays an increasingly more complex iteration of the original TPMS which leaves the fundamental design of the spectrometer largely intact. Thus the TPMS itself is a major continuity of this experimental string in combination with the continuity evidenced in the core group of physicists who proposed the experiments. Most importantly for a physics and detector driven model of social structure, the physics

39 See the Proposal to Study Photoproduction of Final States of Mass Above $2.5 \mathrm{GeV}$ with a Magnetic Spectrometer in the Tagged Photon Lab, submitted October 1, 1976, p 3. The proposal refers to a study performed by $\mathbf{R}$. Rubinstein that supports the feasibility of converting the TPL to a pion beam.

40 The proposal for E-769 (Pion and Kaon Production of Charm and Charmed-Strange States) was submitted to the laboratory in November, 1985. The spectrometer

configuration was so closely based upon the design of the original E-516 configuration, that the collaboration included a diagram of the TPMS in the E-516 configuration (with the recoil detector) and simply explained the differences in the text. The E-791 proposal did not even contain a copy of the spectrometer design, it had become so well known to the community of physicists at Fermilab.

41 A sample of the core group listed on the proposals is Tom Nash (E-516, 691, 769, 791, Fermilab), Jeff Appel (E-516, 691, 769, 791, Fermilab), Jeff Spaulding (E-516, 691, 769, 791, University of Toronto and later Fermilab), Paul Mantsch (E-516, 691, 769, 791, Fermilab), Steve Bracker (E-516, 691, 769, 791, University of Toronto), and Don Summers (E-516, 769, 791, University of California at Santa Barbara and later the University of Mississippi). Another issue that is beyond the scope of this present study is the relationship between the names listed on the formal correspondence of the experiments to the laboratory (proposals etc.), and the actual level of activity of those people over the course of the experiment.

42 Experiment E-769 was approved in December, 1985 and completed in February, 1988. Experiment E-791 was formally submitted to the laboratory as a proposal in November of 1987, approved in June, 1988, and is currently scheduled for a second running period beginning in January of 1991. 
goals of the 4 experiments were already outlined in the original P-516 proposal and reflect a continuing program of measuring the properties of charmed particles using both photoproduction and hadroproduction techniques.

Additional analysis of yet other experiments performed at Fermilab reveals that evidence for the physics and detector driven model and the notion of experimental "strings" is manifested throughout the entire history of the laboratory, spanning the 3 Directorates of Robert Wilson, Leon Lederman, and John Peoples. For example, there are similar continuities revealed in the E-82, 226, 383, 425, 486, 584, $617,731,773$ experimental string which focused its studies upon CP violation with a core group led by Valentine Telegdi and Bruce Winstein. Also, the E-8, 440, 495, 555, $620,619,756$, and 800 experimental string which focussed its studies upon neutral (and later charged) hyperon physics, with a core group led by Lee Pondrum, Gerry Bunce, Ken Heller, Tom Devlin, Kam-Biu Luk, Regina Rameika, and Ken Johns. A final example is the E-21A, 356, 616, and 770 experimental string which focused its studies upon neutrino structure functions and the total neutrino cross section with a core group led by Barry Barish, Frank Sciulli, and Wesley Smith. ${ }^{43}$ My preliminary studies also show that this type of experimental strings are found at other laboratories like CERN. ${ }^{44}$

As I claimed earlier, the complexity of the social structures of such collaborations emerged from the complexity of the spectrometer, which in turn was driven by the complexity of the physics interactions to be detected, in combination with the ability of a core group of physicists to work together effectively as a unit in a continuing program of physics studies that yield successful results. The physics and detector driven model is a heuristic tool with which to extend the descriptive ability to "strings" of experiments. To this point, the physics and detector driven model has provided a basis for understanding the major continuities that link individual experiments into a string of experiments, but it has not provided a framework that fully describes the deeper motivations for such strings. In other words, I have described what the continuities are, but not why these continuities seem to persist through successive iterations of experiments. What are the driving forces that link individual experiments into strings of experiments? Why did this phenomenon emerge at this point in the history of HEP? Can string phenomena be explained within the larger sociological context of laboratory life? More importantly, can they be characterized within the historical context of the development of HEP described in the first section of this paper?

As we already indicated, the history of the development of HEP has been characterized by explicit attempts to remove various types of uncertainties that present themselves in the course of scientific practice. Many of the natural uncertainties involved in intermittent cosmic ray particles and small data samples were removed by the design of accelerators. But this thrust experimenters into new sociological uncertainties that for some have proven even more difficult to navigate, namely the sociological problems involved in proton economics and obtaining experimental real estate. I claim that the phenomenon of experimental strings is yet another instance of this tendency to remove the physics and sociological uncertainties that present themselves within the context of all experiments which

\footnotetext{
43 The examples of such phenomena at Fermilab are too numerous to describe in detail. Other instances are the E-98, 665; E-1A, 310; E-95, 537, 705, 771; E-87, 358, 400, 401, 402, 687; E-497, 715, 761 experimental strings.

44 In regard to photoproduction experiments at CERN during this same time period there were experiments NA1 (1980 run), NA1 (1983 run), and NA14 as well as experiments WA4, WA57, WA58, and WA69. It is beyond the scope of the present work to describe these examples in detail, but this is part of my on-going research in this area.
} 
are performed at a laboratory like Fermilab. In support of this claim, $I$ will once again use the case study of experiments E-516, 691, 769, and 791.

In regard to the removal of physics uncertainties, using the tagged photon beamline for all four experiments meant that the properties of the beamline transport system were well known factors, removing many of the uncertainties of starting up a new beamline. This was true independent of the fact that primary beam was converted from an electron to pion beam. 45 In addition, using the TPMS as a basis for the 4 experiments made the detector a well known entity that could be built upon by the collaboration, thus removing the experimental uncertainties of understanding the systematic errors inherent in shaking down a new spectrometer.46 Because the vast majority of the detector sub-systems remained in tact throughout all 4 configurations of the TPMS, much of the reconstruction software for E-516 could be reused with later experiments with simple modifications to the code. This removed many of the uncertainties involved in writing new code for constantly changing detector geometries. In the case of E-691, the experiment's ability to produce outstanding physics results in a relatively short amount of time following the end of data taking was a direct result of a conscious decision not to modify the majority of the components of the TPMS from the E-516 configuration. 47 Finally, the program of studying photoproduction then hadroproduction of charmed particles, removed some of the uncertainty of what physics goals to propose to the laboratory. Each series of measurements done by one of the experiments was an extrapolation that built upon the previous data and forged ahead into new areas of charmed measurements, accumulating larger and larger samples of charmed events which yielded increasingly precise measurements of the previously unmeasured properties of these particles. 48

In regard to removing the sociological uncertainties, the ability to maintain possession of the TPL for all 4 experiments removed many of the uncertainties inherent in experimental real estate and gaining a position at the end of one of the particle beam spigots. In addition, the fact that the TPMS was utilized with only modifications to the original E-516 design removed many of the uncertainties of obtaining the resources need to build a new spectrometer prior to running an experiment. While the size and complexity of the TPMS drove the original cost of

45 As described earlier, the feasibility of converting the secondary beam from electrons to pions had been establish for almost 10 years prior to E-769.

46 The advantage of minimalizing the systematic errors of the spectrometer was noted as a justification for viewing the TPMS as a "facility" rather than simply as an "experiment" in the original E-516 proposal. See the Proposal to Study Photoproduction of Final States of Mass Above $2.5 \mathrm{GeV}$ with a Magnetic Spectrometer in the Tagged Photon Lab, October 1, $1976, \mathrm{p} 3$.

47 In addition to the evidence of the continuities evidenced in the diagrams of the two experiments, and the statement of this intent in the written text of the E-691 proposal, I confirmed this in a private communication with Rolly Morrison, a member of both experiments.

48 Experiment E-691 accumulated 10,000 fully reconstructed photoproduced charmed cvents and made the most precise measurements of charmed particle lifetimes in the laboratory. E-769 has accumulated 6,000 fully reconstructed hadronically produced charmed events which was also the largest hadronically produced sample of charmed particles produced in the laboratory. Experiment E-791 plans to accumulate 100,000 fully reconstructed hadronically produced charmed particle events during the current running period at Fermilab which will surpass even the samples accumulated by photoproduction mechanisms. 
building the E-516 spectrometer, the TPMS proved to be a good investment of laboratory resources, given the physics results it has produced. The original taxonomizing and distributing of the expertise of the core group institutions around the spectrometer for E-516 removed many of the uncertainties of accumulating the base of expertise needed to carry out the later experiments. Here the continuity of the TPMS itself and the institutions who built it were powerful connections which linked this string of experiments. The continuity of the institutional affiliations also removed much of the uncertainties for the professors and physics departments that needed access to the beams in order to attract top notch students to a productive HEP program and the resources to train them. The importance of the pedagogic function of such experiments ought not be under estimated. In the competitive context of institutionalized HEP, the phenomena of experimental strings removes many of the uncertainties associated with the necessity to publish results regularly and train graduate students. 49 But this experimental string also had a very subtle but real effect upon the Fermilab community's perceptions of the collaboration. With each successive iteration of the TPMS, the collaboration was viewed by their colleagues as a competent group. Their track record at the TPL removed many of the uncertainties about whether they could do what they promised. 50

By systematically removing the above physics and sociological uncertainties, the collaboration also eliminated some of the institutional uncertainties in regard to granting additional resources for each of the experimental progenies. By lowering the "risk factor" associated with the experiment, the laboratory would not only grant them additional resources more easily, but they would also assign them a higher priority than more "risky" experiments in the design, installation, and commissioning phases of each of the subsequent experiments. Lower the laboratory management's "risk factor" had a powerful effect on the sociological factor of "proton economics" for the experiment. When the Directorate and the Physics Advisory Committee (PAC) had to decide how to distribute the fixed number of particles produced by the accelerator, this successful string of experiments which proposed to extend those studies, was more likely to receive the laboratory's support. In fact, at the P-791 proposal presentation to the PAC, the first overhead used by the collaboration had a large picture of a slow-moving turtle on it with a caption that referred to the TPMS as "Old, Slow, and Successful."

In summary, I have claimed that the social structure of experimental strings emerged from 1) the complexity of physics interactions being studied, 2) the complexity of the spectrometer used to detect those interactions, 3) the ability of a core group of physicists to work together effectively as a unit in a continuing program of physics, and 4) as a means of removing many of the physics and sociological uncertainties that are inherent to laboratory life at Fermilab.

\section{Conclusions}

49 Rolly Morrison claims that he was still "putting students through" on the data which he accumulated with E-691 (private communication).

50 The ability to do what they promised was inextricably bound to requests for more and more resources, especially in computing. Thus, while the perception by the HEP community at Fermilab was that they could produce valuable physics results, this heightened the competition with other experiments for laboratory resources. But the ability to "come through" gives an experimental string an advantage over collaborations that cannot appeal to long experimental traditions in support of there requests. 
Using the examples of experiments E-516, 691, 769, and 791, I have tried to argue that a physics and detector driven model is a powerful way to describe the most basic social structures in modern HEP experiments. I also believe that the physics and detector driven model can be extended to large collider experiments like CDF, where a diagram of the detector and a list of the institutions responsible to design and construct the detector sub-systems defines the fundamental structure of the more than 250 physicists in this collaboration. The CDF example also shows that the size and complexity of the detector had necessitated an even more complex higher-order structure be superimposed in order to "manage" the collaboration. With the advent of the next generation of collider detectors like the Solenoidal Detector Collaboration (SDC) and other proposed SSC experiments, physicists must more than ever pay careful attention to the problems posed by the complex social structures of collaborations of 800 physicists, detectors that cost $\$ 700$ million (in 1990 dollars), weigh 10,000 tons, and have 20 million channels of electronics.51 The physics and detector driven model would predict that there would be a correlated increase in the complexity of the social structure of such collaborations - and so there is. Even in the early stage of the Expression of Interest (EOI), the SDC collaboration included an explicit statement of the organization and management of the collaboration, complete with a Governance Document which was ratified by a large majority of the collaboration. 52 But while it may not be clear exactly how such collaborations will function throughout the lifetime of the detector, it is clear that in the absence of a systematic model for organizing such collaborations, that they will probably go on taxonomizing and distributing their expertise along the lines drawn in the diagram of the SDC detector because it is the detector and the physics problems that they know best.

I also tried to argue that the phenomenon of "experimental" strings emerged from factors that can be described partially by the physics and detector driven model, but were also deeply motivated by experimentalists' attempts to avoid the physics and sociological uncertainties produced by the monopoly that laboratory institutions have on the resources for doing HEP. Today, the nuance of the word "string" in HEP is normally associated with the incredibly complex and elegant mathematical formalism that theorists have yet to link to physical effects that are measurable by experiment. But unlike the "theoretical" string theory, the continuities that link individual experiments into "experimental" strings are instantiated both in the detectors and in the collaborations in a definable way. In addition, because some of the experimental strings mentioned above transcend the entire history of Fermilab's research program, and because they seem to emerge so copiously in all areas of HEP research traditions, I believe that the theory of experimental strings, in combination with the recurring attempts of physicists to remove the uncertainties inherent in the institutionalized context of their research, provide a sound sociological and historical foundation for understanding the history of the research program at Fermilab throughout all three of the Fermilab Directorates. 53

51 See the Solenoidal Detector Collaboration Expression of Interest, 24 May, 1990.

52 ibid, p $93 \mathrm{ff}$.

53 This last claim is based upon my on-going research on the history of the physics program in the Lederman era which is being done under the auspices of the Fermilab History Collaboration. 